\section{Estudo \\ Ecidebate}

em Testã⿻

Plamejamento
Revista Estudo \& Debate, Lajeado, v. 26, n. 3, 2019. ISSN 1983-036X

DOI: http://dx.doi.org/10.22410/issn.1983-036X.v26i3a2019.2085

\title{
GESTIÓN DEL CONOCIMIENTO EN EL SECTOR AGRÍCOLA: UN ANÁLISIS BIBLIOMÉTRICO
}

\author{
Lucas Teixeira Costa ${ }^{1}$, Alice Munz Fernandes ${ }^{2}$, Ana Paula Alf Lima Ferreira ${ }^{2}$, \\ Caroline Estefanie do Amaral Brasil Saraiva ${ }^{2}$, Miguel Angel Chinchon Perez ${ }^{1}$
}

\begin{abstract}
Resumen: En los últimos años, la gestión del conocimiento se ha convertido en una alternativa para desarrollar estrategias competitivas y auxiliar en la toma de decisiones en las organizaciones, independiente del sector que participa a fin de obtener ventaja competitiva. En este sentido, la investigación realizada tuvo por objetivo caracterizar las publicaciones científicas que abordan la gestión del conocimiento en el medio agrícola. Para ello, se empleó una investigación cuantitativa descriptiva, operacionalizada mediante análisis bibliométrico. La búsqueda se realizó en la base de datos Scopus, cuya orientación consistió en la Ley de Zipf considerando la ocurrencia de los términos "knowledge management" y "agric*" en el título, resumen y/o palabras-claves. A partir de la inserción de otros criterios de inclusión/exclusión, el portafolio de estudios analizados fueron 230 artículos. Los resultados obtenidos demostraron que en la última década, los estudios que comprenden tal temática se han intensificado. En el área del conocimiento de las publicaciones, se constató que las ciencias agrarias y biológicas abarcan la mayoría de las investigaciones (85\%) y en lo que concierne al origen de autores, China fue destacada (33 autores).
\end{abstract}

Palabras-clave: Agronegocios. Información. Toma de Decisión.

\section{MANAGEMENT OF KNOWLEDGE IN THE AGRICULTURAL SECTOR: A BIBLIOMETRIC ANALYSIS}

\begin{abstract}
In recent years, knowledge management has become an alternative to develop competitive strategies and assist in decision making in organizations, regardless of the sector in order to obtain a competitive advantage. In this sense, the objective of the research was to characterize the scientific publications that approach the knowledge management in the agricultural environment. For this purpose, a descriptive quantitative investigation was carried out using bibliometric analysis. The search was performed in the Scopus database, whose orientation consisted of the Zipf Law considering the occurrence of the terms "knowledge management" and "agric *" in the title, abstract and / or keywords. From the inclusion of other inclusion / exclusion criteria, the portfolio of studies analyzed totaled 230 articles. The results obtained demonstrated that in the last decade, the studies that comprise this theme have intensified. In the area of knowledge of the publications, it was
\end{abstract}

1 Mestrando em Agronegócios pela Universidade Federal do Rio Grande do Sul (CEPAN/UFRGS).

2 Doutoranda em Agronegócios pela Universidade Federal do Rio Grande do Sul (CEPAN/UFRGS). 
verified that the agrarian and biological sciences covered the majority of the investigations (85\%) and as far as the origin of the authors was concerned, China was highlighted (33 authors).

Keywords: Agribusiness. Information. Decision Making.

\section{INTRODUCCIÓN}

A pesar de las transformaciones en todos los contextos de la sociedad prevalecen el papel fundamental de la gestión del conocimiento para el desarrollo socioeconómico de las organizaciones, en el sector agrícola su relevancia todavía es precariamente percibida (GROTTO, 2001). De acuerdo con Dowbor (2001), la intensa gama de datos, informaciones y conocimientos son útiles si no alcanzan al individuo interesado y que tienen potencial para aplicarlo.

Yendo más allá, Bernardo, Binotto y Farina (2017) apuntan que la gestión del conocimiento puede contribuir para la toma de decisión. Sin embargo, para los autores en el ámbito de las cooperativas agrícolas, por ejemplo, las actividades de gestión de conocimiento concernientes a la evaluación, utilización y contribución todavía presentan dificultades. Por ello, la necesidad de mejorar la productividad de los sistemas agroalimentarios a fin de satisfacer la creciente demanda mundial de alimentos y atender la llamada seguridad e inocuidad alimentaria, dirige la atención sobre la gestión del conocimiento disponible (TSCHIEDEL; FERREIRA, 2002).

Sin embargo, Ling (2016) explica que las actividades primarias aún no conocen o invierte en la gestión del conocimiento como mecanismo de eficiencia organizacional para la generación de ventaja competitiva debido a la naturaleza abstracta de tal fenómeno. Es decir, la dificultad de mensuración o visualización inmediata se configura como un obstáculo a la implantación de tal modelo.

En este sentido, la gestión del conocimiento se muestra como una relevante contribución a todos los sectores económicos, incluso en las actividades primarias y de producción agrícola, teniendo en cuenta la significativa participación de dichos agentes en el desarrollo socioconómico mundial. De ahí, se tiene que inicialmente nuevas tecnologías y prácticas de gestión son inicialmente discutidas en ámbito académico y divulgadas a través de publicaciones científicas para posteriormente ser transformadas en mecanismos fácilmente adoptados por la sociedad.

Así, investigaciones dirigidas al análisis de las publicaciones pueden auxiliar en la comprensión de cuán evolucionado y difundido determinado tema está, o aún, cuán interesante es para los investigadores de distintas áreas del conocimiento. Luego, la investigación realizada fue guiada por la siguiente cuestión problema: ¿cuáles son las características de las publicaciones científicas que abordan la temática de gestión del conocimiento en el contexto agrícola?

Con vistas a ello, la investigación realizada tuvo como objetivo caracterizar las publicaciones científicas que abordan la gestión del conocimiento en el contexto agrícola. Para ello, se empleó un análisis bibliométrico en la base de datos Scopus, cuyos descriptores de búsqueda permitió obtener una cartera de artículos en los que se observaron aspectos como año de publicación, periódicos, autores, afiliación y país de origen de éstos. Debido 
a la multidisciplinariedad de los constructos verificados, se consideró también el área de conocimiento en la cual fueron clasificados.

Además de la introducción, este estudio está compuesto por otras cuatro sesiones. La revisión de literatura, donde se presenta aspectos conceptuales acerca de gestión del conocimiento y de la agropecuaria, enfatizando su relevancia y representatividad. El método, concerniente a los procedimientos de colecta y análisis de datos. Consiguiendo, los resultados y las discusiones, contrastándolos con los postulados de las Leyes de la Bibliometría. Y, por último, las consideraciones finales, que circunscriben la investigación y apuntan las limitaciones de ésta y sugerencias para estudios futuros.

\section{REVISIÓN DE LITERATURA}

\subsection{GESTIÓN DEL CONOCIMIENTO}

Debido al advenimiento de la revolución tecnológica y sus avances y transformaciones en el mundo del trabajo, el conocimiento se ha convertido en el principal factor determinante de la generación de riqueza y competitividad. De este modo, sustituye paradigmas basados en patrones tradicio- nales, como tierra, capital y trabajo (BARRETO NETO; MENEZES, 2018), enfatizando la relevancia del capital humano e intelectual (MORAES et al., 2018). Por lo tanto, "este escenario, cada vez más competitivo y en constante mutación, se vuelve imperativo crear, adquirir, renovar, compartir y utilizar el conocimiento en las organizaciones de forma estratégica” (OLIVEIRA et al., 2018, p. 16, traducción propia).

La gestión del conocimiento se está volviendo cada vez más un gran diferencial para las organizaciones, independiente del sector, ya que se configura como un factor determinante para el mantenimiento y éxito de éstas (ZABOT; SILVA, 2002). Así, "para sobrevivir y competir en la 'sociedad del conocimiento', las empresas deben aprender a administrar sus activos intelectuales "(PROBST; RAUB, ROMHARDT, 2002, página 11, traducción propia).

De acuerdo con Alvarenga Neto (2005), la gestión del conocimiento consiste en el conjunto de actividades concernientes a la promoción del conocimiento de la organización, a fin de promover la utilización de las mejores informaciones y conocimientos disponibles con el fin de maximizar la competitividad. Para Clari (2010), identificar y analizar el conocimiento existente, bien como planificar acciones para desarrollarlo junto a la organización, es relevante para la obtención de los resultados deseados. Por lo tanto, se tiene el modelo propuesto por Nonaka y Takeuchi (1997) que objetiva ayudar a las empresas a transformar el conocimiento de sus colaboradores en conocimiento organizacional.

La acumulación del conocimiento tácito en el nivel individual puede ser ampliando a toda organización por medio de los cuatro modos de conversión, formando un espiral del conocimiento, o sea, la interacción del conocimiento tácito y explícito constituye una escala cada vez mayor. El modo de conversión del conocimiento tácito en tácito es denominado socialización. Es el proceso de compartimiento de experiencias directamente de otras personas a partir de la observación e ideas, generando la creación del conocimiento tácito de un individuo a otro (NONAKA; TAKEUCHI, 1997). 
A partir del conocimiento tácito, surge el proceso de externalización, que consiste en la creación del conocimiento en las organizaciones, una vez que se desarrollen nuevos conceptos explícitos a partir del conocimiento tácito. Este por su vez, es compartido por medio de lenguaje hablada o escrita, metáforas, conceptos, conceptos modelos (NONAKA; TAKEUCHI, 1997).

Gaspar (2010) apunta que la conversión del conocimiento tácito en explícito, gana importancia en el momento en que la codificación del conocimiento se vuelve más real. Para el autor, esa conversión ocurre cuando el conocimiento tácito es trasmitido por el proceso de comunicación para la organización, o sea, se vuelve un conocimiento explicito, siendo fácilmente comunicado y entendido por las personas.

A partir de la formalización del conocimiento explicito, éste a su vez, podrá ser en acuerdo con otros conocimientos explícitos y ser diseminado para toda la organización. Sin embargo, el conocimiento explícito generado en la organización puede volver a ser tácito cuando es interpretado y comprendido por un individuo dentro de sus valores y creencias individuales. Esta transformación se denomina internalización, es decir, la formación de opinión a partir de un documento o informe (NONAKA; TAKEUCHI, 1997).

\subsection{SECTOR AGRÍCOLA}

La importancia de la agricultura para el crecimiento de un determinado país se configura como dotado de relevancia en la teoría económica. Los estudiosos clásicos del tema, por ejemplo, ya investigaron los distintos papeles a ser desempeñados por la agricultura en contexto de las economías en desenvolvimiento, como es el caso de Brasil (FIGUEIREDO; BARROS; GUILHOTO, 2005).

El País es reconocidamente competitivo en su producción agrícola (COSTA; GUILHOTO; IMORI, 2013) y el sector ha revelado una mayor capacidad de superar los efectos adversos de las crisis económicas internas e internacionales. Además de estas crisis, otro punto que merece atención en el sector agrícola es la gestión de riesgos, frente a la vulnerabilidad de la actividad agropecuaria frente a riesgos catastróficos, sobre todo aquellos oriundos de fenómenos climáticos adversos (MAPA, 2016).

Figueiredo, Barros y Guilhoto (2005) sintetizan los papeles desempeñados por la agricultura en el proceso de desarrollo económico. El primero es el suministro de materia prima para el desarrollo del sector no agrícola y constituir importante mercado consumidor para los productos industrializados. Además, se tiene la atracción de ganancias cambiarias a través de sus exportaciones, la capacidad de generación de empleo y renta en la economía y, sobre todo, el suministro de alimentos para la población.

Considerando el suministro de alimentos para la población, por la perspectiva de la Organización para la Cooperación y Desarrollo Económico (OCDE, 2017), hasta el 2026, se prevé que la disponibilidad de calorías alcance los 2.450 kcal por día, en media en los países menos desarrollados, y exceda las $3000 \mathrm{kcal}$ por día en los países desarrollados. Así mismo, la inseguridad alimentaria seguirá siendo una preocupación global crucial y la coexistencia de desnutrición suscita nuevos desafíos en muchos países. 


\section{METODOLOGÍA}

La investigación realizada se caracteriza como cuantitativa en relación enfoque del problema y descriptiva en cuanto a la finalidad. Como procedimiento técnico se empleó análisis bibliométrico que consiste en la técnica de mensuración de los factores de producción científica (ARAÚJO, 2006). De acuerdo con Oliveira Lobo (1999), este tipo de investigación proporciona identificar las potencialidades de determinados grupos y / o instituciones de enseñanza, así como realizar un diagnóstico del origen de las publicaciones que componen un conjunto específico de conocimiento científico.

Por ello, la bibliometria es fundamentada en tres leyes básicas, de las cuales son: Ley de Lotka, Ley de Zipf y Ley de Bradford. La primera, también conocida como ley del Cuadrado Inverso, se refiere a la productividad de los autores a través de la relación tamaño frecuencia de estos en un determinado conjunto de manuscrito. La segunda, es conocida como Ley de Mínimo Esfuerzo, que concierne acerca de la frecuencia y / u ocurrencia de aparición de palabras en documentos. Por fin, la tercera es la llamada Ley de la Dispersión, que postula sobre los periódicos en los cuales los artículos fueron publicados (VANTI, 2002).

Por lo tanto, se realizó una búsqueda en la base de datos Scopus, ya que ésta se configura como la mayor fuente de resúmenes y citas literarias revisadas por pares (ELSEVIER, 2017). Como orientación de busca se adoptó la Ley de Zipf considerando la existencia en el título, resumen y / o palabras-claves del término "knowledge management" y de las variaciones concernientes a "agricultural" (agric*). La ocurrencia simultánea de tales términos fue asegurada por la inserción del boleano "and". El tipo de documento analizado fue artículo, cuyo período de publicación correspondió a todos los años hasta la fecha del 15 de septiembre de 2017, es decir, no hubo límite inicial de tiempo.

La Figura 1 presenta la representación esquemática del método de investigación, apuntando los criterios de inclusión de los manuscritos analizados, así como la representatividad de estos en el ámbito de los documentos acerca de la temática abordada. También ilustra la contribución de las Leyes de la bibliometria en análisis de resultado. 
Figura 1 - Representación Esquemática de la Investigación



Fuente: Elaborado por los autores.

Así, mientras que la Ley de Zipf fue empleada para la obtención de los estudios que compusieron el portafolio analizado, las Leyes de Lotka y de Bradford fueron utilizadas para pautar el análisis de los resultados. De este modo, los hallazgos se contrastan con los postulados de tales leyes, a fin de identificar la forma en que estas explican determinadas características de las publicaciones científicas sobre gestión del conocimiento en el contexto agrícola o agropecuario.

\section{ANÁLISIS Y DISCUSIÓN DE LOS RESULTADOS}

De acuerdo con los criterios de búsqueda en la base de datos Scopus se encontraron 512 documentos, 230 de ellos son artículos, los cuales componen el portafolio para análisis de los resultados. Con base en los datos, la primera publicación ocurrió en el año 1973, sin embargo, el tema se mantuvo discreto en las investigaciones empíricas. Después de 17 años de la primera publicación surgieron nuevos estudios. La Figura 2 presenta la distribución temporal de las publicaciones. 
Figura 2 - Distribución temporal de las publicaciones

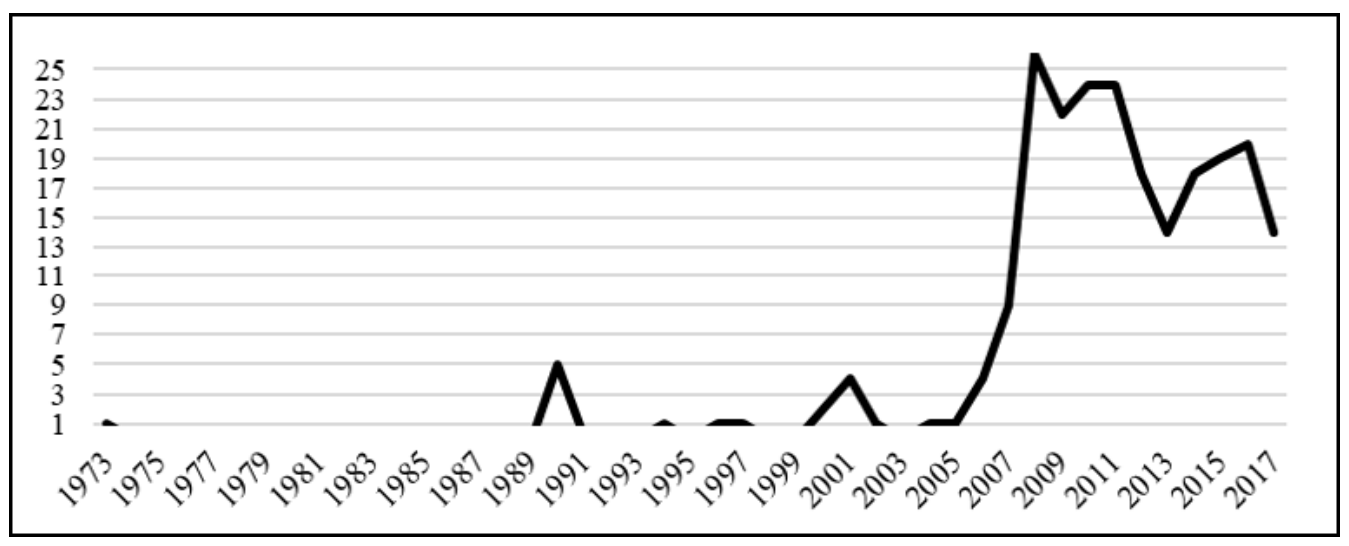

Fuente: Resultado de la investigación (2017).

A partir del 2005 las publicaciones se intensificaron, 2008 fue el año con mayor número de estudios, llegando a 26 artículos $(11,30 \%)$. De este modo, se observó que un número significativo de trabajos fue publicado a partir del año pasado, manteniendo una media de 19,9 artículos por años hasta 2017. Además, los últimos diez años son responsables por $86,52 \%$ de las publicaciones que abordan gestión del conocimiento en el medio agro negocio, denotando el interés creciente por este asunto en el campo científico en la última década.

Las publicaciones se distribuyen en 157 periódicos diferentes, demostrando el alcance y diversidad de fuentes científicas que tienen en su ámbito tal temática, sin embargo, entre ellos, 124 (53,91\%) poseen sólo un artículo publicado. El periódico Computers and Electronics in Agriculture se destaca con mayor número de artículos, posee un total de 14 de documentos $(6,08 \%)$. Tal periódico contempla las áreas de ciencias agrarias y biológicas, con énfasis en horticultura. También destacan otros cuatro periódicos, que son: Nongye Gongcheng Xuebao Transactions of the Chinese Society of Agricultural Engineering (7 artículos); Knowledge Technology (5 artículos); Environmental Science and Policy y Kasetsart Journal Social Sciences, ambos con 4 artículos.

De esta forma, la Ley de Bradford sugiere que los artículos que poseen mayor relevancia en determinada área generalmente son encontrados en un número pequeños de periódicos (HAYASHI et al., 2007), así permite un análisis de nivel de destaque en las áreas del conocimiento científico (GUEDES; BORSCHIER, 2005). Así, los periódicos que demuestran mayor cantidad de publicaciones sobre determinado tema tienden a poseer un núcleo de calidad y relevancia superior en esta referida área del conocimiento (BRADFORD, 1934).

De esta forma, se infiere que entre las publicaciones que abordan la gestión del conocimiento en el campo agro negociador en la base de datos consultada, los artículos que presentan mayor relevancia se encuentran en el periódico con mayor número de publicaciones, o sea, en el periódico Computers and Electronics in Agriculture. 
Cuanto a los autores que abordan la temática en cuestión, se identifica en el portafolio, 157 autores. De estos 124 posen apenas una publicación tratando del tema, el que representa 53,91\% de los artículos. De este modo, el resto de los resultados traen 27 autores con dos artículos cada uno y otros ocho con tres trabajos. Los principales autores son: Bochtis, Feng, Fountas, Lwoga, Nash, Nikkilā, Pesonen e Wiebensohn.

De este modo, se verifica que tales autores representa 10,43\% de las publicaciones, este hecho se encuentra con lo sugerido por la Ley de Lotka en lo que se refiere a la productividad de los autores (VANTI, 2002). De acuerdo con la Ley, se constata que un número reducido de autores poseen "numerosas" publicaciones (MALTRÁS BARBAS, 2003), es decir, se concibe una escala exponencial inversa entre el número de publicaciones científicas por autor (ROUSSEAU; ROUSSEAU, 2000). De esta forma, cuando mayor fuera el número de publicaciones en el área, cada vez más son reconocidos en la misma, adquiriendo mayores las condiciones de perfeccionar sus investigaciones tal (MERTON, 1968).

Referente a las instituciones de afiliación de los autores, se identifican 160 instituciones. Sin embargo, de estas $98(61,25 \%)$ tienen sólo un autor afiliado y, por consiguiente, 42 (26,25\%) instituciones presentan vínculo con dos autores. La Wageningen University and Research Centre obtuvo destaque, apuntada como afiliación de 8 autores, seguida de China Agricultural University e Aarhus Universitet, ambas con 5 autores vinculados. Se encuentran todavía 6 instituciones respondiendo como vínculo de 4 autores, y otras 11 constando como afiliación de 3 autores cada uno. La Figura 3 muestra a las principales instituciones las cuales son autores afiliados.

Figura 3 - Principales instituciones de afiliación

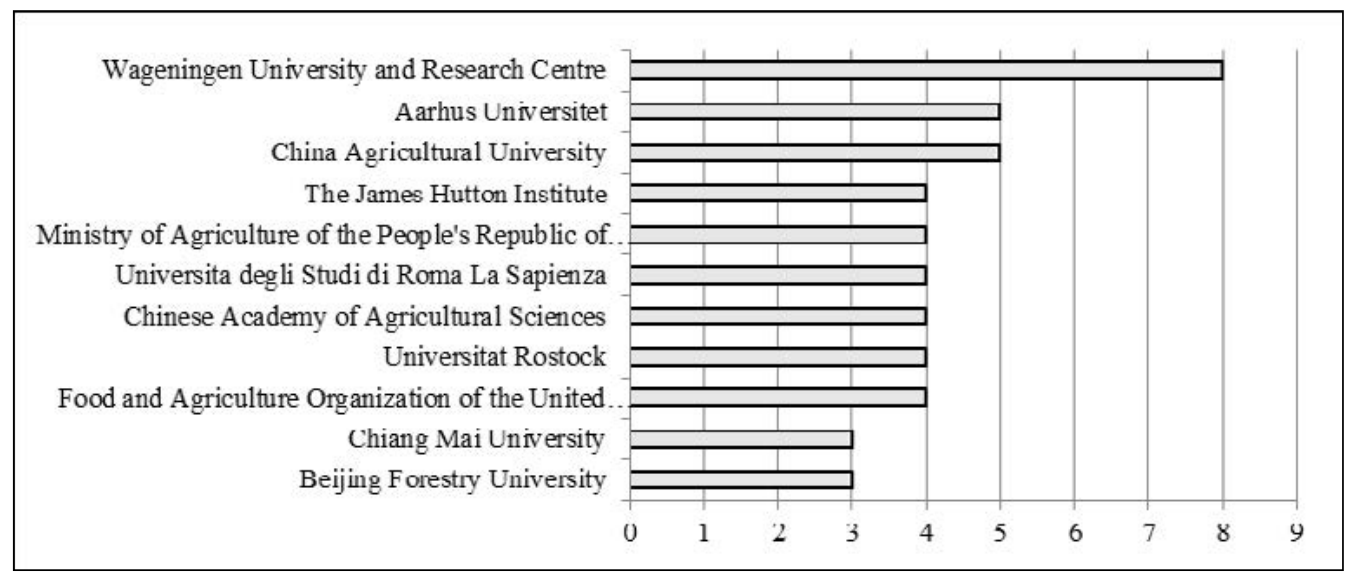

Fuente: Resultados de la investigación (2107).

Con referente al país de origen de los autores, de acuerdo con los resultados, fueron encontradas 73 nacionalidades diferentes. Se destacan los chinos, con 33 autores, seguidos de los americanos con 28, Reino Unido con 22 e Italia con 19 autores. Se enfatiza que Brasil es el $12^{\circ}$ país en el origen de los autores que desarrollan estudios acerca de la gestión 
del conocimiento en el contexto agrícola, indicado por 8 personas. Por lo tanto, la Figura 4 presenta los principales países de origen de los autores.

Figura 4 - Principales países de origen de los autores

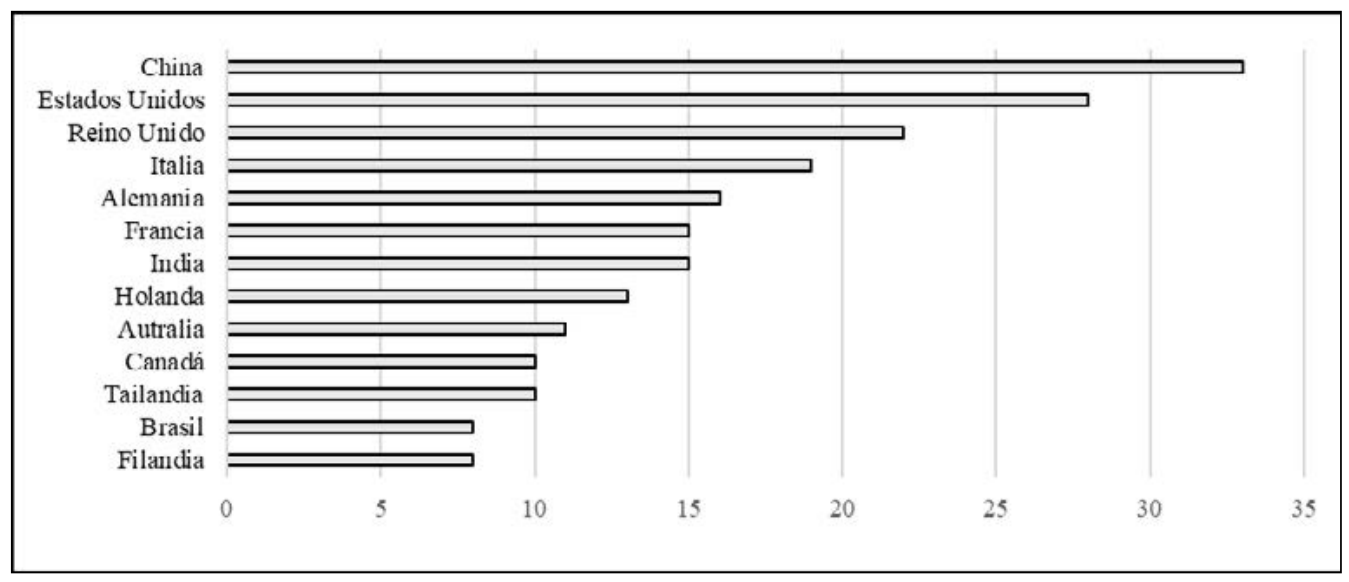

Fuente: Resultados de la investigación (2017.)

Por último, la Figura 5 presenta el área del conocimiento en la que se publicaron los artículos que abordan la temática estudiada. Se resalta que la categoria "otros" compreende las areas cuya presentación individual fue menos que $2 \%$.

Figura 5 - Principales areas de conocimiento

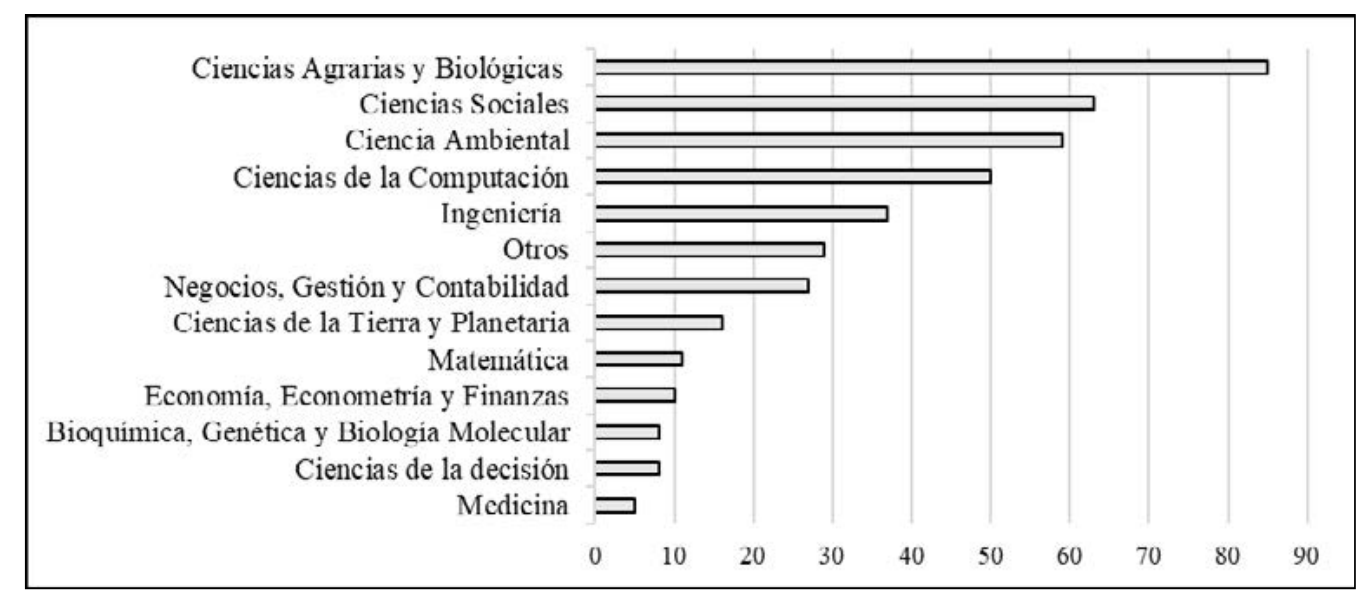

Fuente: Resultados de la investigación.

Se observa que la mayoría (85 artículos) fue publicada contemplando la área de las Ciencias Agrícolas y Biológicas. En este contexto, se puede inferir que tal hallazgo se justifica por la relevancia de la temática como herramienta para la toma de decisión en el medio agrícola. Sin embargo, otras áreas también tuvieron representatividad, tales como: ciencias 
sociales (63 artículos); ciencia ambiental (59 artículos); la ciencia de la computación (50 artículos), entre las demás áreas con menor participación.

\section{CONSIDERACIONES FINALES}

De acuerdo con los resultados obtenidos, se observa que la gestión del conocimiento en el contexto agrícola se configura como una temática abordada en las investigaciones empíricas desde más de cuatro décadas. Sin embargo, en los últimos diez años las publicaciones se han vuelto más frecuentes, lo que puede ser justificado por la percepción de la contribución de tal proceso en la toma de decisión en el medio agro-negociador, objetivando el éxito de las organizaciones.

Se constató que el $86,52 \%$ de los artículos fueron publicados en la última década, denotando la relevancia y el creciente interés por el tema en el ámbito científico. El año 2008 represento $11,30 \%$ del portafolio analizado. Concerniente a los periódicos relacionados, el Computers and Electronics in Agriculture se destacó, con 6,08\% de las publicaciones. En cuanto a los autores, de identifico que 8 autores representan 10,43\% de las publicaciones, de modo que consisten en aquellos dotados de mayor propiedad sobre las temática. Con referencia a las instituciones de investigación a las cuales los autores son afiliados, la Wageningen University and Research Centre obtuvo destaque apuntado como afiliación de 8 autores vinculados.

Se constató también que el país con mayor representatividad en estudios abordados tal temática fue China, correspondiendo a 33 autores. En lo que concierne al área del conocimiento a la que pertenecen las publicaciones, se verifico que las ciencias agrarias y biológicas poseen más estudios relacionados en su ámbito ( 85 artículos). Sin embargo, se reconocen las limitaciones de estudio realizado en cuanto a la utilización de una sola base de datos.

Como sugerencia para investigaciones futuras, se recomienda ampliar la búsqueda en otras bases de datos, a fin de caracterizar de forma más amplia la gestión del conocimiento en las publicaciones científicas relacionadas al medio agrícola. Se sugiere además el uso de otros términos y boleanos, buscando encontrar un foco menor de análisis que permita construir una revisión sistemática de la literatura, con el propósito de profundizar el conocimiento sobre tal temática, corroborando para la identificación de nuevas problemáticas y oportunidad de nuevas investigaciones. También se sugiere analizar el estado del arte sobre la gestión del conocimiento en el sector agrícola, es decir, averiguar de qué forma ambos constructores se conectan con un enfoque analítico y qué tipo de abordaje teórico emplean.

\section{REFERENCIAS}

ALVARENGA NETO, R. C. D. Gestáo do conhecimento em organizaçóes: proposta de mapeamento conceitual integrativo. Tese de Doutorado (Programa de Pós Graduação em Ciência da Informação). Universidade Federal de Minas Gerais. 400f. Belo Horizonte, 2005. 
ARAÚJO, C.A. Bibliometria: evolução histórica e questôes atuais. Em Questáo, v. 12, n. 1, p. 11-32, 2006.

BARRETO NETO, J. F.; MENEZES, A. M. F. O acesso à informação e a difusão do conhecimento no fotalecimento da política de educação profissional da Bahia. Bahia Análise \& Dados, v. 8, n. 1, p. 93-110, 2018.

BERNARDO, L.V.M.; BINOTTO, E.; FARINA, M.J.U.S. Practices of identifying knowledge management in agricultural cooperative. Espacios, v. 38, n. 11, 2017.

BRADFORD, S.C. Sources of information on specific subjects. Engineering, v. 137, p. 85-86, 1934.

CLARI. G.V. A transferência do conhecimento na implantaçáo de um sistema integrado de gestáo para a área de Tecnologia da Informaçáo: Um estudo de caso em uma empresa do setor energético. Dissertação de Mestrado (Programa de Pós-Graduação em Administração de Empresas). Universidade Presbiteriana Mackenzie. 146f. São Paulo, 2010 .

COSTA, C.C.; GUILHOTO, J.J.M.; IMORI, D. Importância dos setores agroindustriais na geração de renda e emprego para a economia brasileira. Revista de Economia e Sociologia, v. 51, n. 4, p. 787-814, 2013.

DOWBOR, L. Tecnologias do conhecimento: os desafios da educação. Petrópolis: Vozes, 2001.

ELSEVIER. About Scopus. Disponível em: < https://www.elsevier.com/solutions/ scopus\&gt>. 2017. Acesso em 15 set. 2017.

FIGUEIREDO, M.G.; BARROS, A.L.M.; GUILHOTO, J.J.M. Relação econômica dos setores agrícolas do Estado do Mato Grosso com os demais setores pertencentes tanto ao Estado quanto ao restante do Brasil. Revista Economia e Sociologia Rural, v. 43, n. 3, p. $557-575,2005$.

GASPAR, M.A. Gestáo do conhecimento em empresas atuantes na indústria de software no Brasil: Um estudo das práticas e suas influências na eficácia empresarial. Tese de Doutorado (Programa de Pós-Graduação em Administração). Universidade de São Paulo. 226f. São Paulo, 2010.

GROTTO, D. Um olhar sobre a gestão do conhecimento. Revista de Ciências da Administraçáo, v. 3, n. 6, p. 31-38, 2001.

GUEDES, V.; BORSCHIVER, S. Bibliometria: uma ferramenta estatística para a gestáo da informação e do conhecimento, em sistemas de informação, de comunicação 
e de avaliação científica e tecnológica. Proceedings CINFORM - Encontro Nacional de Ciência da Informação VI, Salvador -Bahia, 2005.

HAYASHI, M.C.P.I. et al. Um estudo bibliométrico da produção científica sobre a educação jesuítica no Brasil colonial. Biblios, v. 8, n. 27, p. 1-18, 2007.

LING, L.S. Analysis of knowledge management processes for human capital of Malaysian plantation industry. Information (Japan), v. 18, n. 18, p. 3087-3093, 2016.

MALTRÁS BARBA, B. Indicadores de producción. In: MALTRÁS BARBA, B. Los indicadores bibliométricos: fundamentos y aplicación al análisis de la ciência. Gijón: Ediciones Trea, 2003.

MAPA - Ministério da Agricultura, Pecuária e Abastecimento. Plano Agrícola e Pecuário 2016-2017. Secretaria de Política Agrícola. Brasília: Mapa/SPA, 2016.

MERTON, R.K. The Mathew effect Science. Science, v. 159, n. 3810, p. 58, 1968.

MORAES, J. P.; SAGAZ, S. M.; SANTOS, G. L.; LUCIETTO, D. A. Tecnologia da informação, sistemas de informaçôes gerenciais e gestão do conhecimento com vistas à criação de vantagens competitivas: revisão da literatura. Revista Visão, v. 7, n. 1, p. 39$51,2018$.

NONAKA, I.; TAKEUCHI, H. Criaçáo de conhecimento na empresa: como as empresas japonesas geram a dinâmica da inovação. Rio de Janeiro: Campus, 1997.

OECD - Organization for Economic Co-operation and Development. 2017. OECDFAO Agricultural Outlook 2017- 2026. Disponível em: < http://www.oecd-ilibrary.org>. Acesso em: 16 set. 2017.

OLIVERA LOBO, M.D. Métodos y técnicas para la indización y la recuperación de los recursos de la World Wide Web. Boletin de la Asociación Andaluza de Bibliotecários, v. 57, 1999.

OLIVEIRA, M.; SANTOS, E.; CARREIRA, S.; ANDRADE, J. R. A importância da motivação na gestão do conhecimento nas organizaçóes: um estudo numa empresa portuguesa. ISLA Multidisciplinary e-Journal, v. 1, n. 1, p. 16-30, 2018.

PROBST, G.; RAUB, S.; ROMHARDT, K. Gestáo do conhecimento: os elementos construtivos do sucesso. Porto Alegre: Bookman, 2002.

ROUSSEAU, B.; ROUSSEAU, R. Percolation as a model for informetric distributions: fragment size distribution characterized by Bradford curves. Scientometrics, v. 47, p. 195-206, 2000. 
TSCHIEDEL, M.; FERREIRA, M.F. Introdução à agricultura de precisão: conceitos e vantagens. Ciência Rural, v. 32, n. 1, 2002.

VANTI, N.A.P. Da bibliometria à webometria: uma exploração conceitual dos mecanismos utilizados para medir o registro da informaçáo e a difusão do conhecimento. Ciência da Informaçáo, v. 31, n. 2, p. 152-162, 2002.

ZABOT, J.B.M.; SILVA, L.C.M. Gestáo do conhecimento: aprendizagem e tecnologia construindo a inteligência coletiva. Sáo Paulo: Atlas, 2002. 